\title{
Dendrimer functionalized folate-targeted gold nanoparticles for luciferase gene silencing in vitro: A proof of principle study
}

\author{
LONDIWE SIMPHIWE MBATHA \\ FIONA CHEPKOECH MAIYO \\ MOGANAVELLI SINGH* \\ Non-Viral Delivery Laboratory \\ Discipline of Biochemistry \\ University of KwaZulu-Natal \\ Westville Campus \\ School of Life Sciences \\ Private Bag X54001, Durban 4000 \\ South Africa
}

Accepted September 9, 2018

Published online October 28, 2018

\begin{abstract}
Use of exogenous small interfering RNA (siRNA) has shown potential in gene silencing. The need for target-specific siRNA delivery vehicles is crucial to successful gene silencing. This study is aimed at developing and evaluating the safety and efficiency of siRNA delivery using unmodified and folic acid (FA) modified poly(amidoamine) generation 5 (PAMAM G5D) functionalized gold nanoparticles (Au:G5D/Au:G5D:FA) in vitro. All formulations were physico-chemically characterized and nanocomplexes were evaluated using the band shift, dye displacement, nuclease protection, MTT cell viability, and luciferase reporter gene assays. Nanocomplexes bound and protected siRNA against degrading RNases, and were well tolerated by the cells. The Au:G5D:FA nanocomplexes elicited excellent gene silencing in folate receptor expressing HeLa-Tat-Luc cells, decreasing significantly in the presence of excess FA ligand, indicating nanocomplex uptake by the mechanism of receptor mediation. These results highlight the synergistic role played by $\mathrm{Au}$ and the dendrimer in enhancement of transgene silencing.
\end{abstract}

Keywords: siRNA, gold nanoparticles, dendrimers, folic acid, gene silencing

Small interfering RNA (siRNA) has evoked much interest as an effective gene knockdown tool in gene therapy, with potential in treating various incurable disorders such as cancer and acquired immunodeficiency syndrome (AIDS) (1). This gene knockdown ability of siRNA can be accredited to the endogenous elegant RNAi mechanism, discovered by Fire and Mello in the late 1990s, which regulates gene expression by silencing disease inducing genes. Upon delivery of the long, double-stranded RNA (dsRNA) into the cytoplasm of eukaryotic cells, it is subjected to cleavage by the Dicer enzyme into short 21-25 base pair (bp) fragments known as siRNAs. These siRNAs are then loaded into an RNAinduced silencing complex (RISC), triggering RISC activation by the guide strand (antisense) of the siRNA duplex. The activated RISC then cleaves the complementary strand of

\footnotetext{
*Correspondence; e-mail: singhm1@ukzn.ac.za
} 
the target mRNA, resulting in its degradation by intracellular nucleases, and silencing of gene expression (2-3).

Introduction of synthetic siRNA in various mammalian cancer cell lines has been reported to induce the same effect as the inherently produced dsRNA, but the long term gene silencing attained through repeated systemic administrations has raised questions on its potential as a key therapeutic agent. The inability to bypass the cellular membrane due to their hydrophilic and polyanionic nature, and rapid enzymatic degradation, due to the presence of a hydroxyl group at the second carbon atom of the sugar moiety that favors hydrolysis of the phosphodiester backbone, leads to poor gene silencing, which has limited its application to date (4-5). Hence, designing appropriate carriers that are safe and will protect the siRNA from the degrading enzymes while facilitating its targeted uptake by specific cells is a major hurdle in its therapeutic application.

Cationic polymers, such as dendrimers, have been explored as effective pDNA vectors due to their associated high transfection efficiency. The relatively high cytotoxicity of cationic dendrimers, PAMAM in particular, due to nonspecific interactions of primary amine groups with the cell membrane, is the main concern for their potential application in gene therapy studies. In the present study, two approaches have been used to reduce the cytotoxicity of PAMAM dendrimers while preserving their gene delivery efficiency. First, terminal amines of the dendrimer were also modified with gold nanoparticles, forming less toxic NPs. Second, terminal amines of the dendrimers were also partially tailored with a targeting ligand, folic acid, producing folate targeted dendrimer grafted gold nanoparticles (FA:G5D:AuNPs) with reduced toxicity. This study was focused on designing, characterizing and evaluating the cytotoxicity profiles of untargeted and FA targeted PAMAM grafted AuNPs in various mammalian cell lines and their ability to efficiently deliver siRNA to HeLa-Tat-Luc cells to elicit the required gene silencing.

\section{EXPERIMENTAL}

\section{Materials}

Starburst poly(amidoamine), PAMAM dendrimer, generation five (PAMAM G5D), bicinchoninic acid (BCA), folic acid, 1-(3-dimethylaminopropyl)-3-ethylcarbodiimide (EDC), dimethylformamide (DMF), sodium dodecylsulphate (SDS), ribonuclease A (RNase A) and dialysis tubing (MWCO $12000 \mathrm{Da}$ ) were supplied by Sigma-Aldrich (USA). DNA grade agarose was acquired from Bio-Rad Laboratories (USA). Tris(hydroxymethyl)-aminomethane hydrochloride (Tris-HCl), 3-(4,5-dimethylthiazol-2-yl)-2,5-diphenyltetrazolium bromide (MTT), 2-[4-(2-hydroxyethyl)-1-piperazinyl] ethane sulphonic acid (HEPES), dimethyl sulphoxide (DMSO), ethidium bromide (ETB) and gold(III) chloride trihydrate $99 \%\left(\mathrm{HAuCl}_{4}\right)$ were purchased from Merck (Germany). Minimum essential medium (EMEM) containing Earle's salts and L-glutamine, penicillin $\left(500\right.$ units $\left.\mathrm{mL}^{-1}\right)$ /streptomycin $\left(5000 \mu \mathrm{g} \mathrm{mL}^{-1}\right)$ and trypsin-versene were purchased from Lonza-BioWhittaker (USA). Foetal bovine serum (FBS) was provided by Hyclone (USA). Human cells: embryonic kidney (HEK293) was obtained from the American Type Culture Collection (USA), hepatocellular carcinoma (HepG2) and epithelial colorectal adenocarcinoma (Caco-2) cells were purchased from Highveld Biologicals (Pty) Ltd. (South Africa), and HeLa-Tat-Luc was provided by the Department of Physiology (University of KwaZulu-Natal, Durban, South 
Africa). The luciferase assay kit was purchased from the Promega Corporation (USA). The siGENOME non-targeting siRNA (D-001210-01) and anti-Luc siRNA (D-002050-01) [GAUUAUGUCCGGUUAUGUA(UU)] were obtained from Thermo Scientific Dharmacon Products (USA). Duplexes were prepared according to the manufacturer's specifications in $1 \times$ RNA buffer to a final concentration of $20 \mu \mathrm{M}$ and were routinely stored at $-20{ }^{\circ} \mathrm{C}$. All other reagents were of analytical grade and $18 \mathrm{MOhm}$ water was used in reagent preparation, in addition to RNase free water for all siRNA experiments.

\section{Synthesis of gold nanoparticles (AuNPs)}

AuNPs were synthesized by $\mathrm{HAuCl}_{4}$ reduction with trisodium citrate $(1 \%)$ as previously described (6-7). The resultant colloidal solutions were stored for future use and were stable for 6 months.

\section{Synthesis of PAMAM G5D modified folic acid (G5D:FA) conjugate}

PAMAM G5D was modified with FA $\left(\mathrm{C}_{19} \mathrm{H}_{19} \mathrm{~N}_{7} \mathrm{O}_{6}\right)$ through a carbodiimide reaction as previously described (8-9) (Supplementary Fig. S1). Briefly, FA, $2.8 \mu \mathrm{mol}(1.23 \mathrm{mg})$ in $3 \mathrm{~mL}$ of DMF was reacted under $\mathrm{N}_{2}$ with $38.2 \mu \mathrm{mol}(7.3 \mathrm{mg}$ ) of EDC for 45 minutes under constant stirring. This was then added dropwise with stirring into the G5D solution $(3 \mu \mathrm{mol}, 100 \mu \mathrm{L})$ and the $\mathrm{pH}$ was adjusted to 9.5. This solution was stirred for 3 days under nitrogen and then dialyzed against $18 \mathrm{MOhm}$ water for 24 hours to remove excess unreacted byproducts.

Synthesis of dendrimer grafted AuNPs (Au:G5D NPS) and folic acid targeted dendrimer grafted $A u N P_{s}(A u: G 5 D: F A N P s)$

$\mathrm{Au}: G 5 D$ and Au:G5D:FA NPs syntheses were adapted from the Turkevich method to produce a 25:1 gold/dendrimer molar ratio (10). Unmodified G5D and synthesized G5D:FA conjugates were used as templates. All AuNPs were dialyzed as previously described to remove unreacted by-products.

\section{Nanocomplex preparation}

Increasing amounts of NPs ranging from $2-8 \mu \mathrm{g}$ were mixed with either $0.5 \mu \mathrm{g}$ siCONTROL Tox siRNA for binding studies or $0.27 \mu \mathrm{g}$ anti-Luc siRNA for cytotoxicity and transfection studies in $32 \mu \mathrm{L}$ sterile Hepes buffered saline (HBS). This was followed by brief mixing and centrifugation at 13000 revolutions/minute (rpm) for 5 minutes, and a 60 minute incubation at room temperature.

\section{Transmission electron microscopy (TEM) and nanoparticle tracking analysis (NTA)}

A Jeol JEM-1010 transmission electron microscope fitted with a MegaView III digital camera and iTEM UIP software (Japan), set at an acceleration voltage of $200 \mathrm{kV}$, was used to view the morphology of the synthesized nanoparticles and their nanocomplexes prepared at endpoint ratios. NTA (NanoSight NS500; Malvern Instruments Ltd., UK) was used to determine the hydrodynamic diameters and zeta potentials of the nanoparticles and their nanocomplexes at optimum binding ratios. 


\section{Ultra-violet (UV) and proton nuclear magnetic resonance $\left({ }^{1} H\right.$ NMR) spectroscopy}

Attachment of G5D and FA onto the AuNP surface was confirmed using UV-Vis spectroscopy (UV-1650PC, Shimadzu, Japan) and ${ }^{1} \mathrm{H}$ NMR spectroscopy (Bruker DRX 400), using deuterated $\left(\mathrm{D}_{2} \mathrm{O}\right)$ water as a solvent.

\section{Gel retardation assay}

Nanocomplexes at varying $(\mathrm{m} / \mathrm{m}$ ) ratios, containing gel loading buffer ( $50 \%$ glycerol, $0.05 \%$ bromophenol blue, $0.05 \%$ xylene cyanol), were subjected to electrophoresis on a 2 $\%(m / V)$ agarose gel containing ETB $\left(1 \mu \mathrm{g} \mathrm{mL}^{-1}\right)$, in $1 \mathrm{X}$ electrophoresis buffer $\left[36 \mathrm{mmol} \mathrm{L}^{-1}\right.$ Tris- $\mathrm{HCl}, 30$ mmol L-1 sodium phosphate $\left(\mathrm{NaH}_{2} \mathrm{PO}_{4}\right), 10 \mathrm{mmol} \mathrm{L}^{-1}$ ethylenediamine tetraacetic acid (EDTA), pH 7.5], for 1 hour at $50 \mathrm{~V}$. The gels were viewed and images were captured at exposure times of 1-2 seconds using a Vacutec Syngene G: Box BioImaging system (Syngene, UK).

\section{Ethidium bromide intercalation assay}

Compact binding of siRNA to the NPs was assessed fluorescently using a Glomax ${ }^{\circledR}$ Multi+ detection system (Promega), set at an excitation wavelength of $520 \mathrm{~nm}$ and an emission wavelength of $600 \mathrm{~nm}(11)$. ETB $\left(24 \mu \mathrm{L}, 100 \mu \mathrm{g} \mathrm{mL}^{-1}\right)$ was mixed with HBS $(100 \mu \mathrm{L})$ in a 96-well FluorTrac flat bottom black plate and relative fluorescence (RF) was used as baseline $(0 \%)$ fluorescence reading. The $100 \%$ RF was set by introducing $1.3 \mu \mathrm{g}$ siRNA to the mixture, which was followed by systematic addition of $1 \mu \mathrm{L}$ aliquots of the prepared cationic NPs $(0.5 \mu \mathrm{g})$. The RF values were recorded after each addition until a plateau in fluorescence was noticed.

\section{RNase A digestion assay}

Nanocomplexes containing siRNA $(0.5 \mu \mathrm{g})$ and varying amounts of NPs were prepared at three ratios; sub-optimum, optimum, and supra-optimum as determined from the gel retardation assay. Nanocomplexes were exposed to $10 \%$ RNase A for 2 hours at 37 ${ }^{\circ} \mathrm{C}$, followed by addition of EDTA and SDS to final concentrations of $10 \mathrm{mM}$ and $0.5 \%$ $(m / V)$, respectively. After an additional $20 \mathrm{~min}$ incubation at $55^{\circ} \mathrm{C}$, the samples were subjected to electrophoresis as described previously.

\section{Cell viability: MTT assay}

HEK293, HepG2, Caco-2 and HeLa-Tat-Luc cells were trypsinised and seeded into 48well plates at densities of 2.3-2.8 $\times 10^{4}$ cells/well, respectively, and incubated in $0.3 \mathrm{~mL}$ complete medium [EMEM, FBS $(10 \%, V / V)$, penicillin $\mathrm{G}\left(100 \mathrm{U} \mathrm{mL}^{-1}\right)$ and streptomycin sulphate $\left.\left(100 \mu \mathrm{g} \mathrm{mL}{ }^{-1}\right)\right]$ for 24 hours in $5 \% \mathrm{CO}_{2}$ at $37^{\circ} \mathrm{C}$. Prior to addition of the preformed nanocomplexes (containing $0.27 \mathrm{mg}$ siRNA), the medium was replaced with fresh medium $(0.3 \mathrm{~mL})$, and cells were thereafter incubated for 48 hours at $37^{\circ} \mathrm{C}$. The medium was then replaced with $0.3 \mathrm{~mL}$ medium containing $10 \%$ MTT reagent $\left(5 \mathrm{mg} \mathrm{mL}^{-1}\right.$ in sterile PBS), followed by incubation for 4 hours at $37^{\circ} \mathrm{C}$. The medium/MTT mixture was then removed, cells were washed twice with PBS $(0.3 \mathrm{~mL})$, and the resulting purple formazan salt was 
solubilized with $0.3 \mathrm{~mL}$ DMSO. Absorbance values were recorded at $570 \mathrm{~nm}$ in a Mindray MR-96A microplate reader. Percentage cell viability was correlated to untreated cells (control $=100 \%$ ).

\section{Transfection and competition assay}

HeLa-Tat-Luc cells were trypsinized and seeded into 48-well plates at a density of 2.1 $\times 10^{4}$ cells/well and incubated in $0.3 \mathrm{~mL}$ complete medium for 24 hours at $37^{\circ} \mathrm{C}$. Thereafter, the prepared nanocomplexes (containing $0.27 \mathrm{mg}$ siRNA) were added as previously described and the cells were incubated for an additional 48 hours at $37^{\circ} \mathrm{C}$. Cells were then washed with PBS $(2 \times 0.5 \mathrm{~mL})$ and lysed with $80 \mu \mathrm{L} /$ well cell lysis buffer for $15 \mathrm{~min}$ on a Scientific STR 6 platform rocker at $30 \mathrm{rpm}$. Cell lysates were obtained by centrifugation at $12,000 \times \mathrm{g}$ for $1 \mathrm{~min}$. To $20 \mu \mathrm{L}$ of each cell-free extract (supernatant), $100 \mu \mathrm{L}$ of luciferase assay reagent was added, mixed and luminescence was measured in relative light units (RLU) on a Glomax ${ }^{\circledR}$-Multi+Detection System (Promega Biosystem, USA). Protein concentrations of cell-free extracts were determined using the standard BCA assay, and the luciferase activity was expressed as RLU/mg protein.

For competition studies, $50 \mathrm{mM}$ of folic acid solution was incubated with the cells for $20 \mathrm{~min}$ at $37^{\circ} \mathrm{C}$ prior to the addition of nanocomplexes. Thereafter, the luciferase activity and protein concentration were determined as above.

\section{Statistical analysis}

Cell viability and transfection studies were performed in triplicate and the results were expressed as means \pm standard deviation (SD). Experimental data was analyzed by two-way ANOVA and $t$-test, using GraphPad Prism 6.0 and statistical significant values are indicated by ${ }^{*} p<0.05, \# p>0.05,{ }^{* *} p<0.01$, and ${ }^{* * *} p<0.001$.

\section{RESULTS AND DISCUSSION}

\section{Morphology, size and zeta potential of nanoparticles and nanocomplexes}

Dendrimer functionalization of AuNPs is a promising advancement in the design of efficient non-viral delivery vectors (12). Conjugation of G5D onto AuNPs, as well as attachment of FA moieties onto G5D, were verified using UV (Fig. S2, Supplementary) and ${ }^{1} \mathrm{H}$ NMR spectroscopy (Fig. S3-4, Supplementary). The surface plasmon resonance (SPR) peak at $536 \mathrm{~nm}$ indicated the formation of AuNPs, with the shift to a longer wavelength of 566 nm suggesting a modification of the AuNP surface due to the attachment of G5D. The absorption peak at $287 \mathrm{~nm}$ for Au:G5D:FA correlates with that of FA, suggesting successful attachment of the FA moiety (13-14). In ${ }^{1} \mathrm{H}$ NMR spectroscopy, which was confirmed from literature, the chemical shift between $2.25-3.34 \mathrm{ppm}$, representing the amino $\left(\mathrm{NH}_{2}\right)$ and methylene $\left(\mathrm{CH}_{2}\right)$ proton peaks of $\mathrm{G} 5 \mathrm{D}$, and the characteristic proton peaks between 6.50 $8.63 \mathrm{ppm}$ indicating the conjugation of FA moiety were observed (15).

TEM (Fig. 1) and NTA (Table I) revealed spherical NPs with diameters ranging from 65-128 nm, while their nanocomplexes appeared as globular clusters with diameters ranging from $124.9-162.9 \mathrm{~nm}$. There were no significant differences $(p<0.05)$ in the mean sizes 
L. S. Mbatha et al:: Dendrimer functionalized folate-targeted gold nanoparticles for luciferase gene silencing in vitro: A proof of principle study, Acta Pharm. 69 (2019) 49-61.

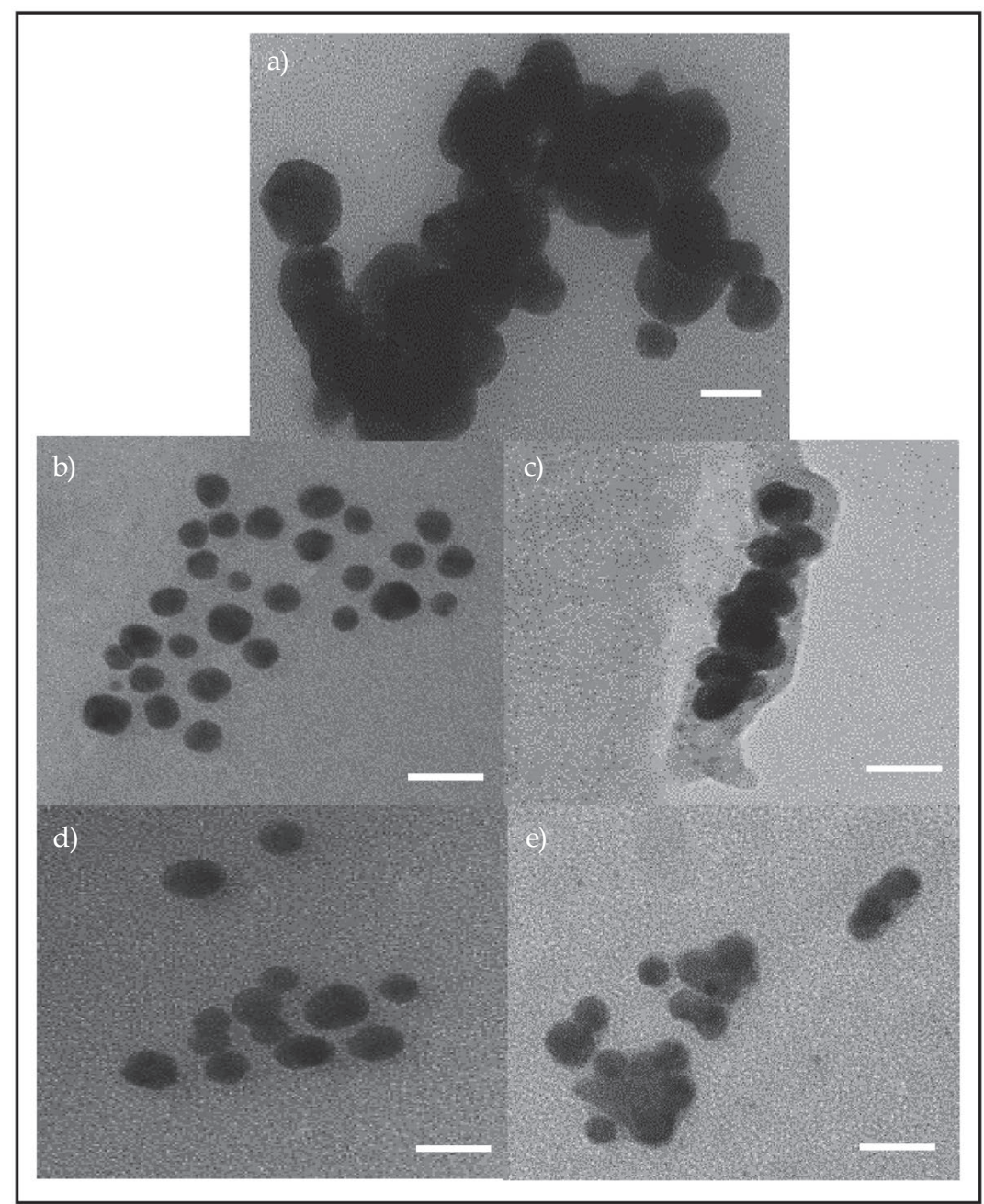

Fig. 1. TEM micrographs of: a) AuNPs, b) Au:G5D, c) Au:G5D-siRNA, d) Au:G5D:FA and e) Au:G5D:FAsiRNA. Nanocomplexes prepared at the optimum weight ratio of 5.0:1 and 8.0:1 ( $\mathrm{m} / \mathrm{m})$, respectively. Scale bar $=100 \mathrm{~nm}$.

observed when test nanocomplexes (Au:G5D and Au:G5D:FA) were compared with control nanocomplexes (G5D and G5D:FA). Furthermore, both the NPs and their nanocomplexes

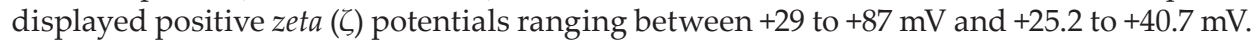
These $\zeta$ potential measurements are greater than $+25 \mathrm{mV}$ and are hence associated with relatively high colloidal stability (16). Au:G5D and Au:G5D:FA nanocomplexes exhibited greater stability than the G5D and G5D:FA nanocomplexes, as noted by their significantly higher $\zeta$ potentials. Reduced $\zeta$ potentials of the nanocomplexes may also infer little shielding of positive charges of the dendrimers by the folate moieties. 
L. S. Mbatha et al:: Dendrimer functionalized folate-targeted gold nanoparticles for luciferase gene silencing in vitro: A proof of principle study, Acta Pharm. 69 (2019) 49-61.

Table I. Mean size and $\zeta$ potential measurements of nano-scaffolds and their nanocomplexes. Data are presented as mean diameter or $\zeta$ potential $\pm S D$.

\begin{tabular}{lccc}
\hline $\begin{array}{l}\text { Nanoparticles and } \\
\text { nanocomplexes }\end{array}$ & $\begin{array}{c}\text { NP:siRNA }(m / m) \\
\text { ratio }\end{array}$ & $\begin{array}{c}\text { Mean diameter } \pm \text { SD } \\
(\mathrm{nm})\end{array}$ & $\begin{array}{c}\zeta \text { Potential } \pm \text { SD } \\
(\mathrm{mV})\end{array}$ \\
\hline $\mathrm{Au}$ & - & $65.90 \pm 9.80$ & $-7.3 \pm 1.6$ \\
$\mathrm{G} 5 \mathrm{D}$ & - & $161.3 \pm 11.9$ & $+87.2 \pm 2.4$ \\
$\mathrm{Au}: G 5 \mathrm{D}$ & - & $100.5 \pm 44.1$ & $+20.9 \pm 2.2$ \\
G5D:FA & - & $128.0 \pm 1.20$ & $+71.2 \pm 3.4$ \\
$\mathrm{Au}: G 5 D: F A$ & - & $77.70 \pm 12.5$ & $+29.0 \pm 0.5$ \\
Au:G5D-siRNA & $5.0: 1$ & $124.9 \pm 19.9^{\sharp}$ & $+38.5 \pm 2.7^{*}$ \\
Au:G5D:FA-siRNA & $8.0: 1$ & $162.9 \pm 51.9^{\sharp}$ & $+40.7 \pm 1.3 \#$ \\
G5D-siRNA & $5.0: 1$ & $154.9 \pm 9.50^{\sharp}$ & $+25.8 \pm 0.8^{*}$ \\
G5D:FA-siRNA & $6.0: 1$ & $149.7 \pm 5.70^{\sharp}$ & $+37.2 \pm 1.3 \#$ \\
\hline
\end{tabular}

${ }^{*} p<0.05,{ }^{*} p>0.05$ when test nanocomplexes are compared with control nanocomplexes. NTA size and zeta potential distribution are reflected in supplementary notes.

\section{Gel retardation assay}

This assay demonstrated the ability of the prepared NPs to bind and complex the siRNA. This is based on the principle that was initially reported by Hellman and Fried (17) that during electrophoresis, nucleic acids migrate freely across the agarose gel, but when

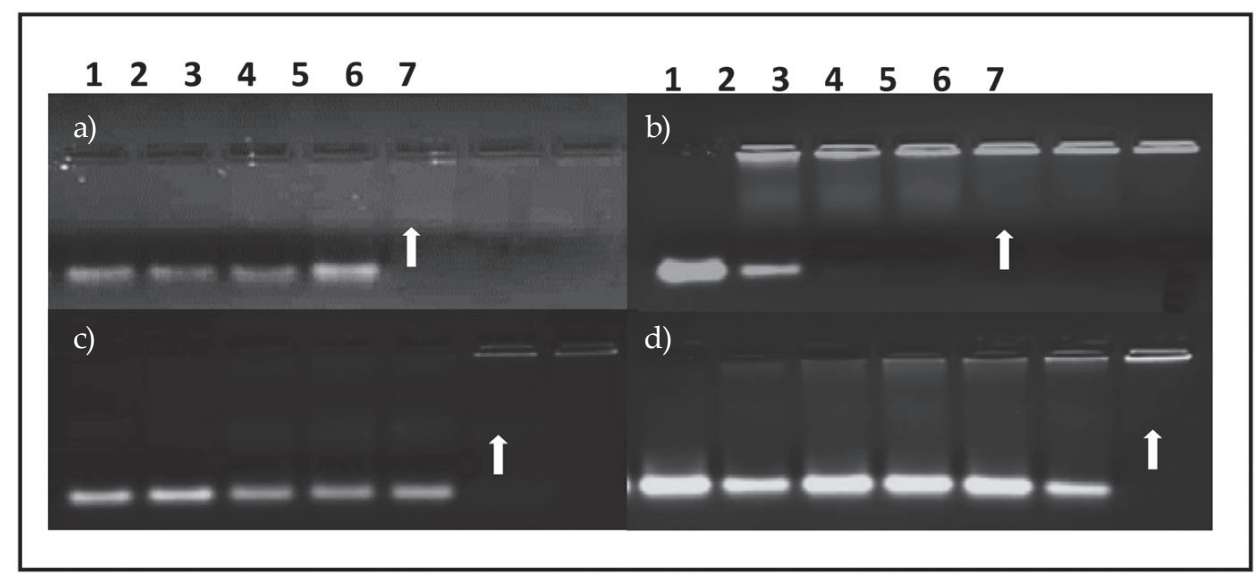

Fig. 2. Gel retardation assay showing the binding of: a) G5D, b) Au:G5D, c) G5D:FA, d) Au:G5D:FA and siRNA. Nanocomplexes contained varying amounts of nanoparticles and $0.5 \mu \mathrm{g}$ siCONTROL Tox siRNA corresponding to weight ratios of 2:1, 3:1, 4:1, 5:1, 6:1, and 7:1 in lanes 2-7, respectively (a-c); and 3:1, 4:1, 5:1, 6:1, 7:1, and 8:1 in lanes 2-7, respectively (d). Lane 1: naked siRNA. Arrows indicate endpoint ratios. 


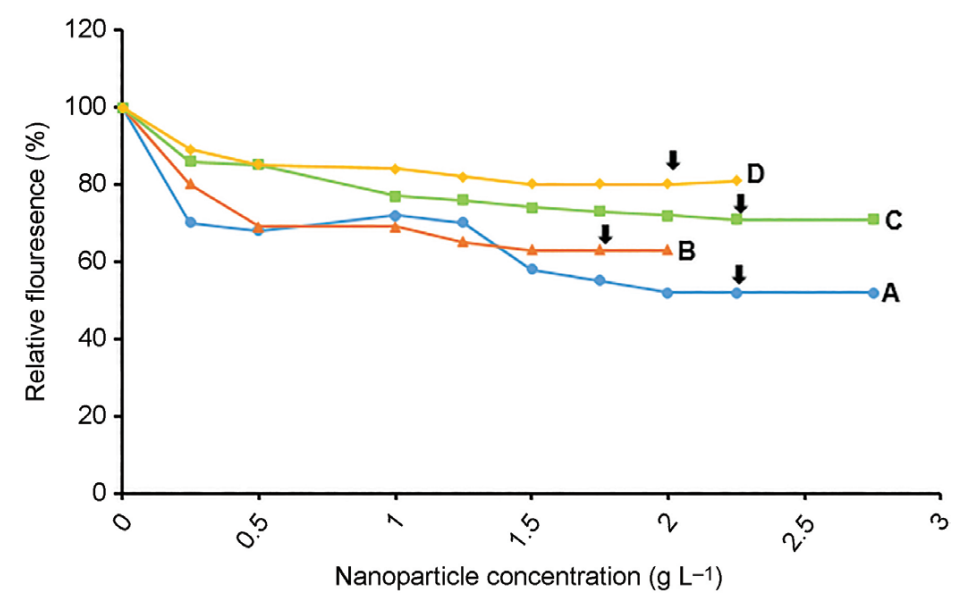

Fig. 3. Ethidium bromide displacement assay of (A) G5D, (B) Au:G5D, (C) G5D:FA, and (D) Au:G5D:FA nanoparticles. Arrows indicate the point of complexation.

they are complexed with carriers, their mobility is retarded. Fig. 2 confirms that all prepared NPs were capable of binding and complexing siRNA. Untargeted G5D and Au:G5D NPs were both completely retarded at a $\mathrm{m} / \mathrm{m}$ ratio of 5:1, while the targeted G5D:FA and Au:G5D:FA NPs were completely retarded at $m / m$ ratios of $6: 1$ and $8: 1$, respectively. The high binding ratios for G5D:FA and Au:G5D:FA NPs may be attributed to some masking of the cationic charges on G5D by folic acid. FA containing nanocomplexes displayed higher positive $\zeta$ potentials with little decrease in positive charge, as evidenced by the $\zeta$ potential measurements.

\section{Ethidium bromide intercalating assay}

The degree of siRNA compaction was explored by measuring the decrease in ETB fluorescence upon its displacement from siRNA by NP induced condensation $(3,11)$. In Fig. 3 , a steady decrease in fluorescence was noted upon stepwise addition of the NPs until a plateau was reached where no further condensation occurred. G5D and Au:G5D nanocomplexes attained this endpoint at 55 and $62 \%$, respectively, while the G5D:FA and Au:G5D:FA nanocomplexes reached the endpoint at 70 and $78 \%$, respectively. A higher degree of compaction is indicative of weaker binding, and suggests the possibility of easier dissociation of siRNA during transfection (18).

\section{RNase A digestion assay}

Naked siRNA delivery to a target site may be compromised by degrading serum nucleases. Hence, delivery vectors that will not only bind but also protect the siRNA against such enzymes are vital. Following exposure to $10 \%$ RNase A, all NPs showed the ability to protect the siRNA across the range of tested ratios (Fig. 4). This was not the case of the uncomplexed/naked siRNA, as indicated by the absence of a band due to total degradation by RNase. 


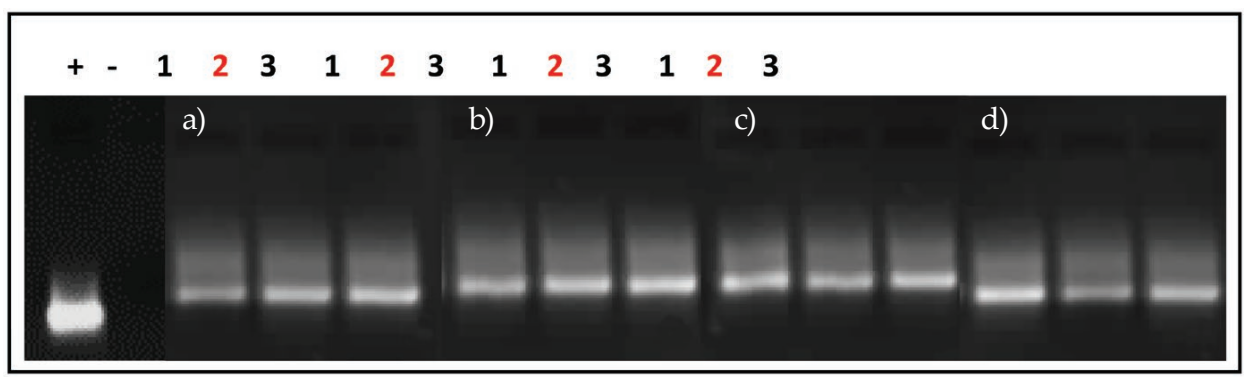

Fig. 4. RNase A digestion of nanocomplexes: a) G5D, b) Au:G5D, c) G5D:FA, d) Au:G5D:FA. Control: naked siCONTROL Tox siRNA $(0.5 \mu \mathrm{g})$ in the absence (+ means positive control) or presence (- means negative control) of RNase A. Lanes 1-3 NP:siRNA ratios: a) 4:1, 5:1, 6:1; b) 4:1, 5:1, 6:1; c) 5:1, 6:1, 7:1; d) 7:1, 8:1, 9:1 ( $\mathrm{m} / \mathrm{m})$. Red numbers indicate optimum binding ratios.

\section{MTT assay}

Biomedical applications of NPs as gene delivery agents often involve deliberate, direct injection or ingestion into the body. These NPs are often coated with bio-conjugates such as nucleic acids, polymers, antibodies and proteins for specific cell targeting (19). Hence, it is crucial to ensure that such enhancements are not detrimental to the cells. Therefore, in vitro toxicity evaluation of NPs is an important aspect to consider when assessing their in vivo potential.

The results presented in Fig. 5, show some cell specific cytotoxicity, indicated by the differences in the $\%$ cell numbers and the level of cytotoxicity. There was no significant

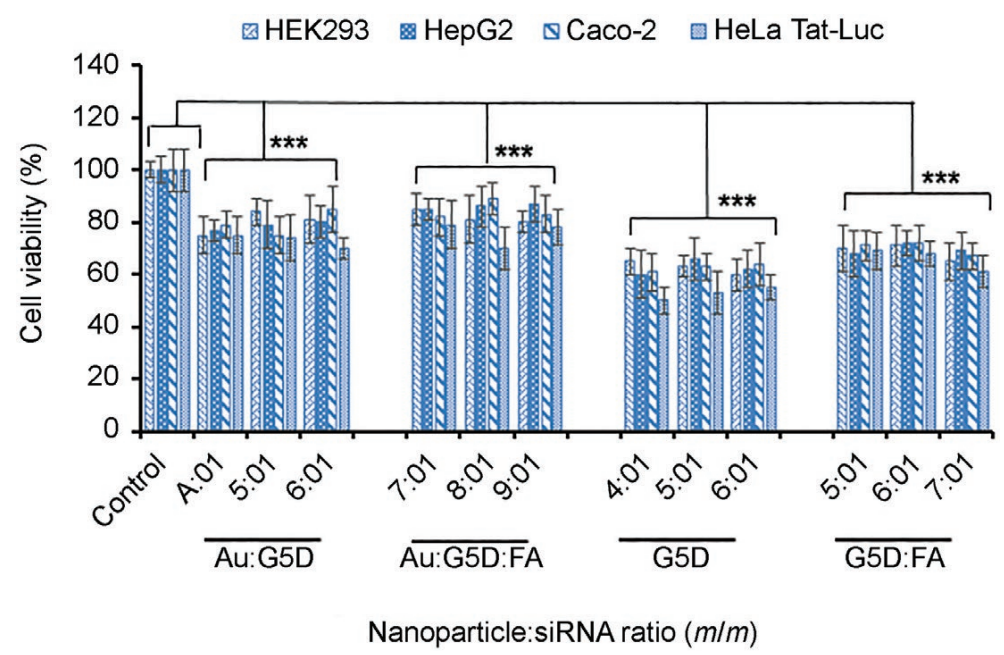

Fig. 5. MTT assay of the NP:siRNA nanocomplexes in HEK293, HepG2, Caco-2, and HeLa-Tat-Luc cells. Data are presented as means \pm SD $(n=3)$. Control $=$ untreated cells. ${ }^{* * *} p<0.0001$ when compared to the control. 
difference $(p>0.05)$ in cell viability between the tested cell lines; however, when compared to the control (cells only), a significant difference $(p<0.0001)$ in cell viability was seen. Higher cell viabilities observed with the Au:G5D and Au:G5D:FA nanocomplexes, ranging from $70-90 \%$, suggest that they were less toxic than the G5D and G5D:FA nanocomplexes (50-70\%). This could be due to the presence of the biocompatible, non-immunogenic and non-cytotoxic AuNPs (supplementary Fig. S5), which reduced some of the amines of G5D (20-22), thereby reducing the cytotoxicity. Hence, lower cell viability for the G5D and G5D:FA nanocomplexes might be due to the increased cationic charges on the G5D even after inclusion of a targeting moiety, folic acid. These findings strongly suggest that the inclusion of non-toxic, inert AuNPs in the formulation of these NPs had a positive and favourable influence on cellular toxicity.

\section{Transfection and competition assay}

This study evaluated the ability of the nanocomplexes to efficiently deliver siRNA to the folate receptor positive HeLa-Tat-Luc cells, a human cervical cell line that stably expresses the firefly luciferase gene (23). The anti-Luc siRNA, which targets the firefly luciferase mRNA, was used to determine the level of gene silencing.

Experimental data revealed that all nanocomplexes were internalized by the selected cells, either by adsorptive non-specific or specific endocytotic pathways, depending on the formulation (14). Lower gene silencing efficiency (34\%) was seen with naked siRNA, as expected, since upon delivery into the cell, naked nucleic acids such as siRNA are subjected to enzymatic degradation (24). Gene silencing elicited by all nanocomplexes ranged from 42-70 \%, with that of the Au:G5D:siRNA and Au:G5D:FA:siRNA nanocomplexes ranging between 50-70 \%, while that for the G5D and G5D:FA nanocomplexes ranged between $42-51 \%$ (Fig. 6). The weaker gene silencing observed for the G5D and G5D:FA nanocomplexes could be due to poor dissociation between siRNA and cationic G5D due to their strong binding affinity. Early studies have demonstrated a direct correlation between the binding affinity of nucleic acids with cationic polymers and transgene expression (25). These findings corroborate the studies conducted by Kang and co-workers, who associated unmodified and conjugated G5D with weak gene silencing efficiency (26). The highly significant gene silencing efficiency elicited by the Au:G5D:siRNA and Au:G5D:FA nanocomplexes can be accredited to the presence of AuNPs entrapped in the $1^{\circ}$ amines, which have been reported to help preserve the morphological aesthetics of dendrimers, permitting efficient interaction with siRNA (21).

These findings are in agreement with the reports by Kolhatkar and coworkers on surface tailored PAMAM showing good cell membrane permeability and good delivery of siRNA into the targeted site (27). Recent studies have also demonstrated the effectiveness of internally modified and surface tailored G5D in gene silencing $(21,28)$. The highest gene silencing was observed at optimum ratios for all nanocomplexes. These findings correlate with previous reports suggesting a possible dependency of the siRNA-dendrimer complex's gene silencing ability on the dendrimer generation, siRNA concentration and binding ratio (29).

To confirm that the uptake of the folate-targeted nanocomplexes was receptor-mediated, a competition study was conducted where the FA-Rs overexpressed on the surface of HeLa-Tat-Luc cells were blocked with an excess of free folic acid prior to transfection with 


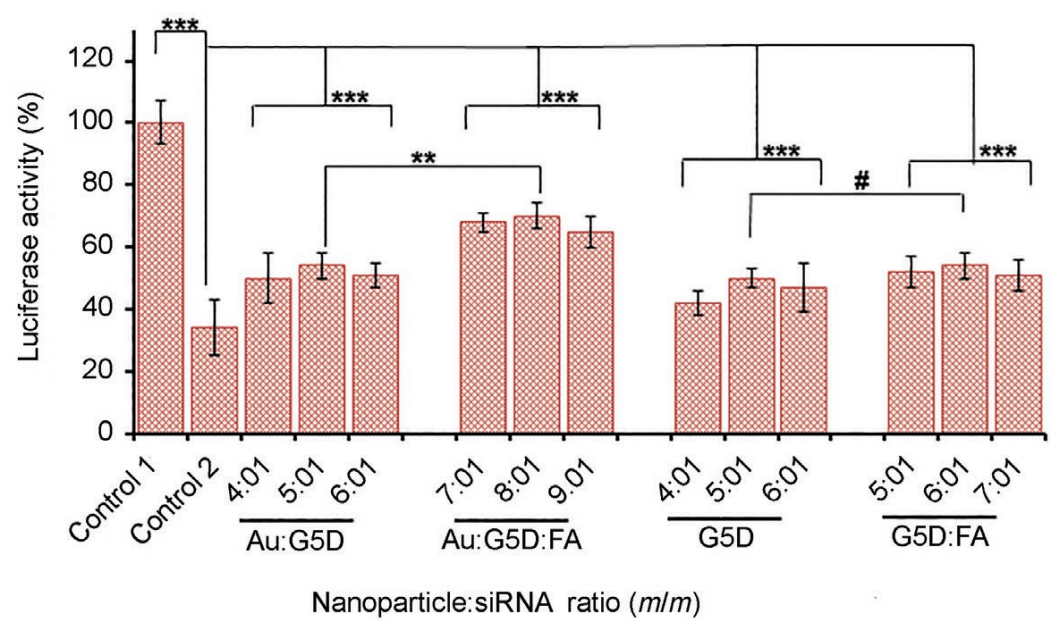

Fig. 6. Luciferase gene silencing by anti-luc siRNA nanocomplexes. Control $1=$ untreated cells. Control $2=$ cells + Anti-Luc siRNA. Data are presented as means \pm SD $(n=3) .{ }^{*} p>0.05,{ }^{* *} p<0.001^{* * *} p<0.0001$.

the nanocomplexes. A significant $(p<0.0001) 30 \%$ drop in gene silencing was seen (Fig. 7) indicating that many of these nanocomplexes were endocytosed via FA-Rs (30). Overall, these findings suggest that the Au:G5D and Au:G5D:FA NPs appear to be better siRNA delivery vehicles than G5D and G5D:FA:siRNA NPs, a finding that correlates with the cell viability studies.

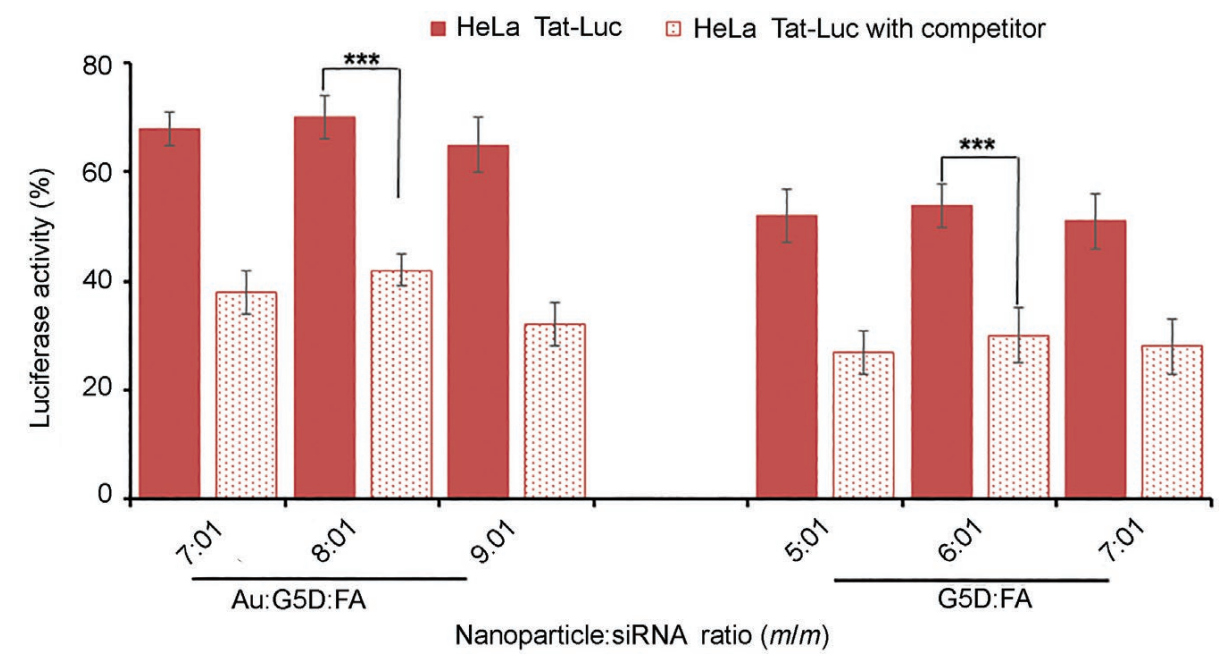

Fig. 7. Receptor competition studies of FA targeted anti-luc siRNA nanocomplexes. Luciferase gene silencing is expressed/reported as percentage. Data are presented as means $\pm \mathrm{SD}(n=3)$. ${ }^{*} p<0.05$, ${ }^{* *} p<0.001,{ }^{* * *} p<0.0001$. 


\section{CONCLUSIONS}

Both Au:G5D and Au:G5D:FA NPs were excellent siRNA delivery vehicles, forming stable globular nanocomplexes that displayed favourable interaction with siRNA and afforded good protection. Modification of surface amines of the dendrimers with the FA targeting ligand and the internal modification with AuNPs served to produce a reduction in cytotoxicity of the nanocomplexes (cell viability of up to $90 \%$ ) and increased siRNA induced transgene silencing (up to $75 \%$ ). In summary, we have demonstrated that Au:G5D and Au:G5D:FA NPs have many of the ideal characteristics required for an efficient siRNA delivery vehicle, and future studies are needed to evaluate these nanocomplexes in vivo.

Acknowledgement. - The authors acknowledge the National Research Foundation, South Africa, (M. Singh, grant ID:81289) for funding. Suplemmentary material available upon request.

\section{REFERENCES}

1. Z. Ziraksaz, A. Nomani, M. Soleimani, B. Bakhshandeh, E. Arefian, I. Haririan and M. Tabbakhian, Evaluation of cationic dendrimer and lipid as transfection reagents of short RNAs for stem cell modification, Int. J. Pharm. 448 (2013) 231-238; https://doi.org/10.1016/j.ijpharm.2013.03.035

2. Y.-C. Tseng, S. Mozumdar and L. Huang, Lipid-based systemic delivery of siRNA, Adv. Drug Deliv. Rev. 61 (2009) 721-731; https://doi.org/10.1016/j.addr.2009.03.003

3. S. Dorasamy, N. Narainpersad, M. Singh and M. Ariatt, Novel targeted liposomes deliver sirna to hepatocellular carcinoma cells in vitro, Chem. Biol. Drug Des. 80 (2012) 647-656; https://doi. org/10.1111/j.1747-0285.2012.01446.x

4. M. Banan and N. Puri, The ins and outs of RNAi in mammalian cells, Curr. Pharm. Biotechnol. 5 (2004) 441-450; https://doi.org/10.2174/1389201043376643

5. D. J. Gary, N. Puri and Y.-Y. Won, Polymer-based siRNA delivery: perspectives on the fundamental and phenomenological distinctions from polymer-based DNA delivery, J. Control. Rel. 121 (2007) 64-73; https://doi:10.1016/j.jconrel.2007.05.021

6. J. Turkevich, P. C. Stevenson and J. Hillier, A study of the nucleation and growth processes in the synthesis of colloidal gold, Discuss. Faraday Soc. 11 (1951) 55-75; https://doi:10.1039/DF9511100055

7. G. G. Lazarus, N. Revaprasadu, J. López-Viota and M. Singh, The electrokinetic characterization of gold nanoparticles, functionalized with cationic functional groups, and its interaction with DNA, Colloids Surf. B. 121 (2014) 425-431; https://doi.org/10.1016/j.colsurfb.2014.06.032

8. E. C. Wiener, S. Konda, A. Shadron, M. Brechbiel and O. Gansow, Targeting dendrimer-chelates to tumors and tumor cells expressing the high-affinity folate receptor, Invest. radiol. 32 (1997) 748-754; https://doi.org/10.1097/00004424-199712000-00005

9. Y. Wang, R. Guo, X. Cao, M. Shen and X. Shi, Encapsulation of 2-methoxyestradiol within multifunctional poly (amidoamine) dendrimers for targeted cancer therapy, Biomaterials 32 (2011) 33223329; https://doi.org/10.1016/j.biomaterials.2010.12.060

10. X. Shi, K. Sun and J. R. Baker Jr, Spontaneous formation of functionalized dendrimer-stabilized gold nanoparticles, J. Phys. Chem. C. 112 (2009) 8251-8258; https://doi.org/10.1021/jp801293a

11. C. T. de Ilarduya, Y. Sun and N. Düzgüneş, Gene delivery by lipoplexes and polyplexes, Eur. J. Pharm. Sci. 40 (2010) 159-170; https://doi.org/10.1016/j.ejps.2010.03.019

12. T. Xiao, X. Cao and X. Shi, Dendrimer-entrapped gold nanoparticles modified with folic acid for targeted gene delivery applications, J. Control. Release 172 (2013) e114-e115; https://doi.org/10.1016/j. jconrel.2013.08.275

13. D. Pan, J. L. Turner and K. L. Wooley. Folic acid-conjugated nanostructured materials designed for cancer cell targeting, Chem. Commun. (2003) 2400-2401; https://doi.org/10.1039/B307878G 
L. S. Mbatha et al:: Dendrimer functionalized folate-targeted gold nanoparticles for luciferase gene silencing in vitro: A proof of principle study, Acta Pharm. 69 (2019) 49-61.

14. G. A. Mansoori, K. S. Brandenburg and A. Shakeri-Zadeh, A comparative study of two folateconjugated gold nanoparticles for cancer nanotechnology applications, Cancers 2 (2010) 1911-1928; https://doi.org/10.3390/cancers2041911

15. Y. Chang, N. Liu, L. Chen, X. Meng, Y. Liu, Y. Li and J. Wang, Synthesis and characterization of DOX-conjugated dendrimer-modified magnetic iron oxide conjugates for magnetic resonance imaging, targeting, and drug delivery, J. Mater. Chem. 22 (2012) 9594-9601; https://doi.org/10.1039/ C2JM16792A

16. S. Honary and F. Zahir, Effect of zeta potential on the properties of nano-drug delivery systems-a review (Part 2), Trop. J. Pharm. Res. 12 (2013) 265-273; http://dx.doi.org/10.4314/tjpr.v12i2.19

17. L. M. Hellman and M. G. Fried, Electrophoretic mobility shift assay (EMSA) for detecting proteinnucleic acid interactions, Nat. Protoc. 2 (2007) 1849-1861; https://doi.org/10.1038/nprot.2007.249

18. C.-C. Chuang and C.-W. Chang, Complexation of bioreducible cationic polymers with gold nanoparticles for improving stability in serum and application on nonviral gene delivery, ACS Appl. Mater. Inter. 7 (2015) 7724-7731; https://doi.org/10.1021/acsami.5b00732

19. N. Lewinski, V. Colvin and R. Drezek. Cytotoxicity of nanoparticles, Smal. 4 (2008) 26-49; https:// doi.org/10.1002/smll.200700595

20. R. Shukla, V. Bansal, M. Chaudhary, A. Basu, R. R. Bhonde and M. Sastry, Biocompatibility of gold nanoparticles and their endocytotic fate inside the cellular compartment: a microscopic overview, Langmuir 21 (2005) 10644-10654; https://doi.org/10.1021/la0513712

21. Y. Shan, T. Luo, C. Peng, R. Sheng, A. Cao, X. Cao, M. Shen, R. Guo, H. Tomás and X. Shi, Gene delivery using dendrimer-entrapped gold nanoparticles as nonviral vectors, Biomaterials 33 (2012) 3025-3035; https://doi.org/10.1016/j.biomaterials.2011.12.045

22. S. H. Lee, K. H. Bae, S. H. Kim, K. R. Lee and T. G. Park, Amine-functionalized gold nanoparticles as non-cytotoxic and efficient intracellular siRNA delivery carriers, Int. J. Pharm. 364 (2008) 94-101; https://doi.org/10.1016/j.ijpharm.2008.07.027

23. A. Daniels, M. Singh and M. Ariatti, Pegylated and non-pegylated siRNA lipoplexes formulated with cholesteryl cytofectins promote efficient Luciferase knockdown in HeLa tat luc cells, Nucleos. Nucleot. Nucl. 32 (2013) 206-220; https://doi.org/10.1080/15257770.2013.776078

24. M. Elsabahy, A. Nazarali and M. Foldvari, Non-viral nucleic acid delivery: key challenges and future directions, Curr. Drug Deliv. 8 (2011) 235-244; https://doi.org/10.2174/156720111795256174

25. T. Bettinger, R. C. Carlisle, M. L. Read, M. Ogris and L. W. Seymour, Peptide-mediated RNA delivery: a novel approach for enhanced transfection of primary and post-mitotic cells, Nucleic Acids Res. 29 (2001) 3882-3891; https://doi.org/10.1093/nar/29.18.3882

26. H. Kang, R. DeLong, M. H. Fisher and R. L. Juliano, Tat-conjugated PAMAM dendrimers as delivery agents for antisense and siRNA oligonucleotides, Pharm. Res. 22 (2005) 2099-2106; https://doi. org/10.1007/s11095-005-8330-5

27. R. B. Kolhatkar, K. M. Kitchens, P. W. Swaan and H. Ghandehari, Surface acetylation of polyamidoamine (PAMAM) dendrimers decreases cytotoxicity while maintaining membrane permeability, Bioconjug. Chem. 18 (2007) 2054-2060; https://doi.org/10.1021/bc0603889

28. B. Weide, S. Pascolo, B. Scheel, E. Derhovanessian, A. Pflugfelder, T. K. Eigentler, G. Pawelec, I. Hoerr, H.-G. Rammensee and C. Garbe, Direct injection of protamine-protected mRNA: results of a phase 1/2 vaccination trial in metastatic melanoma patients, J. Immunother. 32 (2009) 498-507; https://doi.org/10.1097/CJI.0b013e3181a00068

29. M. L. Patil, M. Zhang, S. Betigeri, O. Taratula, H. He and T. Minko, Surface-modified and internally cationic polyamidoamine dendrimers for efficient siRNA delivery, Bioconjug. Chem. 19 (2008) 1396-1403; https://doi.org/10.1021/bc8000722

30. S. Zhang, H. Gao and G. Bao, Physical principles of nanoparticle cellular endocytosis, ACS Nano 9 (2015) 8655-8671; https://doi.org/10.1021/acsnano.5b03184 